\title{
TRACING MOLECULAR EMISSION IN SPIRAL GALAX IES: THE NEAR INFRARED CORRESPONDENCE
}

\author{
R. L. HURT, J. L. TURNER, \& D. LEVINE \\ UCLA Dept. of Astronomy, Los Angeles, CA, USA \\ K. M. MERRILL, I. GATLEY \\ NOAO, Tucson, AZ, USA
}

\section{INTRODUCTION}

Near infrared imaging can be a powerful tool in tracing the densest molecular structures in galaxies. The observable molecular emission originates in large molecular cloud complexes which are also subject to significant extinctions caused by the associated dust. It can be difficult to distinguish between regions of moderate and large molecular density with optical observations as both will appear optically thick. Since extinction in the near infrared is only about a tenth of the corresponding visual extinction, multi-band near infrared imaging will trace the regions of the highest optical depths much more effectively. With the advent of large format infrared imaging arrays it is now possible to use infrared extinction maps as a probe of the large scale distribution of molecular emission in extragalactic sources.

\section{FIGURES}

We present ratio maps between the $\mathrm{J}$ and $\mathrm{K}$ bands for the galaxies IC 342, Maffei 2 , NGC 1068, \& M83. These images trace infrared extinction from the nucleus out into the spiral arms. Darker regions represent higher infrared extinction as $\mathrm{J}$ band will suffer fractionally more from dust extinction than will $\mathrm{K}$ band $\left(A_{V}=\lambda^{-1.8}\right.$ for V-K bands; Draine \& Lee 1988, $A p J, 285,89$ ). Noise begins to dominate the images where the infrared intensities are low. The second image in each set overlays a contour plot of the molecular emission on the extinction map.

The correspondance between the $\mathrm{CO}$ and near IR extinction is striking in these images. The $2^{\prime}$ of emission in IC 342 (Levine, Turner, \& Hurt 1993, in preparation) is traced closely by the IR extinction. The ${ }^{13} \mathrm{CO}(1-0)$ emission in Maffei 2 which traces molecular gas density (Hurt \& Turner 1991, ApJ, 377, 434) follows the variations seen in extinction with remarkably high fidelity. The ring and nuclear gas seen in NGC 1068 have extinction analogues, although the nucleus of this seyfert has extinction far in excess of the corresponding molecular gas (Planesas, Scoville, \& Myers 1991, ApJ, 368, 364). The extinction seen in M83 indicates that the molecular ridge extends well beyond this recent $O$ wens Valley mosiac (Turner, Hurt, \& Kenney 1993, in preparation). 
IC 342

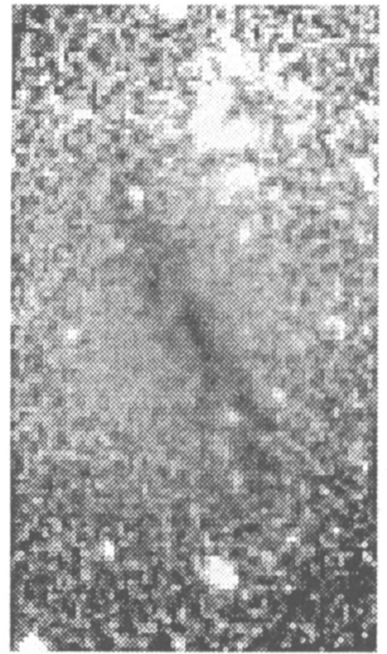

\section{NGC 1068}

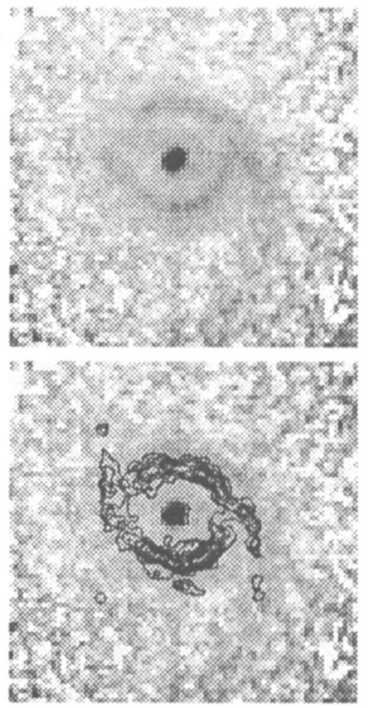

MAFFEI 2
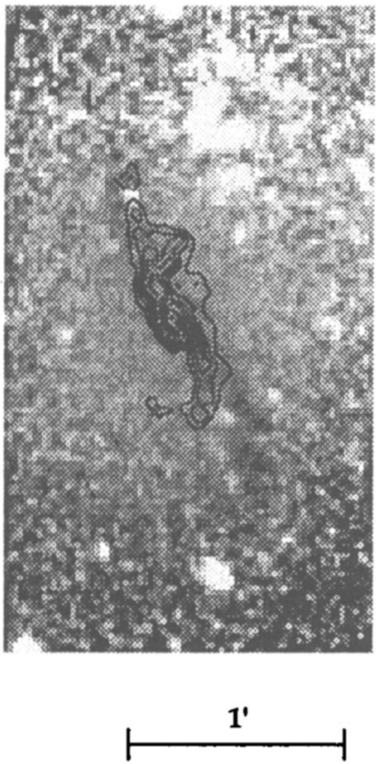

\section{M83}

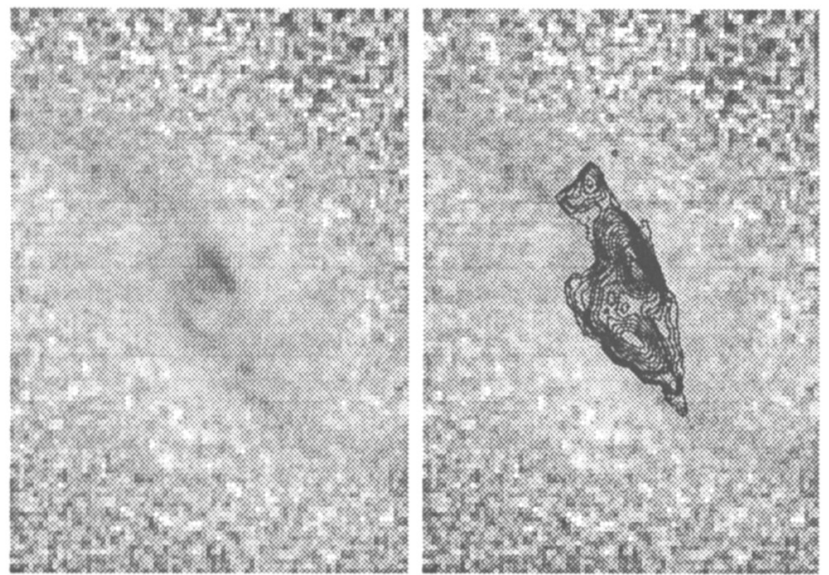

FIGURE 1: INFRARED EXTINCTION AND MOLECULAR GAS $\mathrm{J} / \mathrm{K}$ Ratios (greyscale) with integrated CO emission (contours) 